www.jmscr.igmpublication.org

Impact Factor (SJIF): 6.379

Index Copernicus Value: 71.58

ISSN (e)-2347-176x ISSN (p) 2455-0450

crossref DOI: https://dx.doi.org/10.18535/jmscr/v6i5.128

Journal Of Medical Science And Clinical Research

IGM Publication

An official Publication of IGM Publication

\title{
Fungal Otitis Externa: Clinical and Fungal Evaluation in Hyderabad- Karnataka Region
}

\author{
Authors \\ Channabasawaraj. B. Nandyal ${ }^{1}$, Ramchandra Joshi ${ }^{2}$, Quasser $^{3}$, Sahana $^{4}$, Pallavi $^{5}$ \\ ${ }^{1}$ Professor and Head, ${ }^{2,3,4,5}$ Junior Residents \\ Department of ENT, M. R. Medical College, Kalaburagi, Karnataka, India
}

\begin{abstract}
Background: Ear is a paired structure and a specialized sense organ for hearing and balance. Fungal otitis externa is a superficial fungal infection of the external auditory canal causing lot of suffering to the patient which is commonly seen in ENT practice. Even though the condition is worldwide in distribution is more common in humid tropical and sub-tropical regions like Hyderabad-Karnataka Region, as the climatic condition predispose to fungal otitis externa. Hence, this present study is taken to evaluate the predisposing factors and common fungal isolates seen in patients with fungal otitis externa in Hyderabad-Karnataka region

Aims and Objective: To evaluate the Predisposing factors and common fungal isolates seen in patients with fungal otitis externa in Hyderabad-Karnataka region.

Material and Methods: The present study was conducted on 160 clinically diagnosed cases of Fungal Otitis Externa attending the ENT OPD for a period of one year. Ear discharge specimens were collected on two sterile cotton swabs. Direct Examination of the specimen was carried out by Gram stain and 10\% KOH Preparation. All specimens were inoculated on Sabouraud's Dextrose Agar. Identification of fungi was done as per standard protocol.

Results: Out of 160 patients Evaluated, Fungal isolates were found in 138 cases (86.25\%). The highest incidence was noted in the age group of 31-40 years, more prevalent in Females (60.86\%), with unilateral distribution more on right ear. Pain in the ear was most commonest symptom (95.65\%) followed by itching (92.75\%) and Most common fungi isolated were Aspergillus Flavus, Aspergillus Niger and Aspergillus Fumigatus followed by Candida Species.

Conclusion: It is concluded from our study that Fungal otitis externa is one of commonest conditions seen with prevalence varying with climatic conditions, but warm humid environments support their growth, and the human ear canal is ideal for their proliferation. It occurs in men and women of all ages and is usually a unilateral disease. Aspergilli were considered as predominant fungi for fungal otitis externa. The study also concludes that patients have to be educated not to use unsterile materials to clean the ears which will be effective in prevention of fungal otitis externa.
\end{abstract}

Keywords: Aspergillus, Candida, External Auditory Canal, Fungal Otitis Externa, Otalgia.

\section{Introduction}

Fungal otitis externa is a common condition encountered in a general otolaryngology clinic setting and its prevalence in this study is $7 \%$ among patients who presented with signs and symptoms of otitis externa. It is almost in accordance with other studies. ${ }^{1}$ It is a pathologic entity, with candida and aspergillus the most 
common fungal species. ${ }^{2.3}$ It is not clear that the fungi are the true infective agents or mere colonization species as a result of compromised local host immunity secondary to bacterial infection. Various predisposing factors include a humid climate, presence of cerumen, instrumentation of the ear, increased use of topical antibiotics/steroid preparations ${ }^{4}$, immune compromised host, patients who have undergone open cavity mastoidectomy and those who wear hearing aids with occlusive ear mold. The infection is usually unilateral and characterized by inflammatory itching, scaling and otalgia. Sometimes fungal otitis externa presents as a challenging disease in presence of otorrhoea, for its long-term treatment and follow up, yet its recurrence rate remains high\& so thorough evaluation is required.

\section{Materials and Methods}

The present study was carried out on 160 clinically diagnosed cases of fungal otitis externa attending the ENT OPD at Basaveshwar Teaching and General Hospital Gulbarga. The study was conducted over a period of one year. Ethical clearance was obtained from the Institutional Ethical Committee. An informed consent was obtained from all the patients before the start of study.

Inclusion Criteria: Patients diagnosed with fungal otitis externa based on Symptoms like Otalgia, Itching, aural fullness, Otorrhea and Impaired hearing with Otoscopic findings like appearance of cotton woolly debris/foul smelling discharge, wet blotting paper appearance or creamy white debris.

Exclusion Criteria: Patients on oral \&topical antibiotic \& antifungal drops and negative fungal isolates by direct microscopy or culture were excluded from the study.

Collection of Sample: After clinical diagnosis of Fungal otitis externa was made, all the cases were evaluated for fungal isolates by taking with sterile cotton swabs from fungal like debris seen in the infected ear canal and sent immediately to microbiology laboratory and subjected for $10 \%$ Potassium Hydroxide (KOH) Preparation, Grams Stain Examination \& Sabouraud's Dextrose Agar (SDA) inoculated media were incubated at room temperature and were followed for two weeks. For isolation of fungi growth on media were confirmed by Lacto phenol cotton blue (LCB) preparation and Grams Stain.

\section{Results}

Out of 160 clinically diagnosed cases of Fungal otitis externa only 138 cases produced positive fungal isolates, these constituted $86.25 \%$ which were taken up for further studies. Highest incidence was noted in the age group of 31-40 years $(28.1 \%)$.In our study it has been found that the incidence of Fungal otitis externa was higher in females 84 cases $(60.86 \%)$ than in males 54 cases $(39.1 \%)$ with laterality to right ear.As depicted in Table 1 it was observed in our study that most common predisposing factor was use of unsterile materials/swabs for cleaning the ear in 106 cases $(76.81 \%)$. The use of unsterile materials varied from Safety pins, Hairpins, Matchsticks, Pens and Refills to Unsterile buds followed by use of oils/ mixture of oils 102 cases $(73.91 \%)$.

Table 1: Predisposing factors for fungal otitis externa

\begin{tabular}{|l|c|c|}
\hline Predisposing factors & $\begin{array}{c}\text { Number of } \\
\text { cases }\end{array}$ & Percentage \\
\hline $\begin{array}{l}\text { Use of unsterile sticks/ } \\
\text { ear buds for cleaning } \\
\text { the ear }\end{array}$ & 106 & 76.81 \\
\hline $\begin{array}{l}\text { Use of oils/mixture of } \\
\text { oils }\end{array}$ & 102 & 73.91 \\
\hline $\begin{array}{l}\text { Use of customary head } \\
\text { cover or turban }\end{array}$ & 48 & 30 \\
\hline Nothing suggestive & 8 & 12.80 \\
\hline
\end{tabular}

Pain in the ear was the commonest symptom in 132 cases $(95.65 \%)$ followed by itching in the ear in 128 cases $(92.75 \%)$, the other symptoms observed are depicted in Table 2 
Table 2: Clinical presentation of fungal otitis externa:

\begin{tabular}{|l|c|c|}
\hline Symptoms & Number of Cases & Percentage \\
\hline Pain & 132 & 95.65 \\
\hline Itching in ear & 128 & 92.75 \\
\hline $\begin{array}{l}\text { Aural fullness/ } \\
\text { impaired hearing }\end{array}$ & 87 & 63.04 \\
\hline Otorrhoea & 46 & 33.33 \\
\hline
\end{tabular}

Fungal isolates encountered in our study is shown in Table 3. Aspergillus were the most common fungi isolated in 127 cases of which 81 were Aspergillus Flavus, 40 were Aspergillus Niger and 06 were Aspergillus Fumigatus, followed by Candida albicans in 08 cases.

Table 3: Fungal isolates in fungal otitis externa

\begin{tabular}{|l|c|c|}
\hline Fungal isolates & Number of Cases & Percentage \\
\hline Aspergillus niger & 40 & 28.98 \\
\hline Aspergillus flavus & 81 & 58.69 \\
\hline $\begin{array}{l}\text { Aspergillus } \\
\text { fumigatus }\end{array}$ & 06 & 4.34 \\
\hline Candida albicans & 08 & 5.79 \\
\hline Candida species & 01 & 0.72 \\
\hline Mucor species & 01 & 0.72 \\
\hline Penicillium & 01 & 0.72 \\
\hline Total & 138 & 100 \\
\hline
\end{tabular}

\section{Discussion}

Fungal otitis externa is a superficial mycotic infection of the outer ear canal frequently encountered by otolaryngologist and can usually be diagnosed by clinical examination. However, the correct diagnosis requires a high index of suspicion and thorough evaluation. The infection may be either subacute or acute and is characterized by inflammation, itching, scaling and severe discomfort. The mycosis results in inflammation, superficial epithelial masses of cotton woolly debris containing hyphae or wet blotting paper appearance, suppuration and pain. In addition, symptoms of hearing loss and aural fullness are as a result of accumulation of fungal debris in the canal. Aspergillus and Candida species are the most commonly identified fungal pathogens in fungal otitis externa. ${ }^{5,6}$ Infections with Candida can be more difficult to detect clinically with otorrhoea because of its lack of a characteristic appearance as compared to its appearance of wet blotting paper without otorrhoea and high degree of suspicion of candidial infection is to be suspected when otorrhoeais not responding to aural antimicrobial. ${ }^{7}$ Fungal otitis externa attributed to Candida is often identified by culture data. In this study, we found that fungal otitis externa is more common in females $(60.86 \%)$ ), than in males (39.14\%). Similar results were observed in several other studies ${ }^{8} \mathrm{We}$ found that fungal otitis externa was more common in adolescents and young adults between ages $11-40$ years. We had $46 \%$ of the cases in patients of 11-40 years of age, which was similar to the results obtained by Kaur et al ${ }^{9}$. The occurrence of bilateral fungal otitis externa is very low ${ }^{10}$. In our study it was commonly seen in right ear, which almost correlates with Paulose K et $\mathrm{al}^{11}$. Use of unsterile sticks and swabs for cleaning the Ear and use of oils and mixture of oils in Ear were the commonest predisposing factors as per our study. These results are in accordance with the study done by Mohanty ${ }^{12}$ and Prasad $\mathrm{SC}^{13}$. Unsterile sticks may cause trauma (usually minor and hence unnoticed) for the skin of external auditory canal and deposition of fungi in the wound leading to fungal infection ${ }^{14}$.Fungal study shows mixed presence of fungi ${ }^{15}$ but the most common fungal isolate determined in our study belonged to the Taxon Aspergillus followed by Candida, Mucor and Pencilium. Among the Aspergillus, Aspergillus Flavus was the commonest isolate followed by Aspergillus Niger and Aspergillus Fumigatus. The moisture, warmth and change in acidic pH of the External Auditory Canal provide ideal growth requirements for the Fungi. Aspergilli have an optimum $\mathrm{pH}$ range of 5.7 at a temperature of 37 and this is conducive for all species of Aspergillus. Our observations regarding fungal isolates are in accordance with earlier studies ${ }^{16}$.

\section{Conclusion}

In our evaluation, it is concluded that the disease to be more common in females. The major predisposing factors being Trauma to the External 
Auditory Canal and use of unsterile oil. Otalgia and Itching are the commonest symptoms. Aspergilli and Candida were the predominant Fungi isolated, as these fungi survive on exfoliated dead epithelial cells along with humid climate in External Auditory Canal. The study also concludes that Patients have to be educated not to use unsterile materials to clean the Ears which will be effective in prevention of Fungal otitis externa. Clinical suspicion should always be put on Mycological confirmation especially with otorrhoea to prevent bothersome complications and morbidity of Patients.

\section{References}

1. Mugliston T, O’Donoghue G. Fungal otitis externa: a continuing problem. J Laryngol Otol. 1985;99(4):327-333

2. Kaur R, Mittal N, Kakkar M, Aggarwal AK, Mathur MD. Fungal otitis externa; a clinicomycologic study. Ear Nose Throat J. 2000;79(8):606-960.

3. VennevaldI, Schonlebe J, Klemm E. Mycological andhistological investigations in Humans with middle ear infections. Mycoses. 2003;46(1-2):12-18.

4. Meirtusovas ,Simaljakova M. Yeast and fungi isolated at the mycology laboratory of the First Dermatology Clinic of the Medical Faculty Hospital of Comenens University in Bratislava (1995-2000) Epidermoil Microbial Immunal.2003;52:76-80.

5. Sood VP, Sinha A, Mohapatra LN. Fungal otitis externa; a clinical entity-clinical and experimental study. J Laryngol Otol. 1967;81 (9):999-1004.

6. Youssef YA, Abdou MH. Studies on fungus infection of the external ear Mycological and clinical observations. J Laryngol Otol. 1967; 81 (4):401-412.

7. Youssef YA, Abdou MH. Studies on fungus infection of the external ear Mycological and clinical observations. J Laryngol Otol. 1967; 81 (4):1005-1012.
8. Barati B, Okhovvat SA, Goljanian A, et al. Fungal otitis externa in central Iran: a clinical and mycological study. Iranian Red Crescent Medical Journal 2011;13(12):873-876.

9. Kaur R, Mittal N, Kakkar M, et al. Fungal otitis externa: a clinicomycologic study. Ear, Nose and Throat Journal 2000;79(8):606-609.

10. Zaror L, Fischman O, Suzuki F, et al. Fungal otitis externa in São Paulo. Revista do Instituto de Medicina Tropical de São Paulo 1991;33(3):169-173.

11. Paulose KO, Al Khalifa S, Shenoy P, Sharma RK. Mycotic infection of the ear (fungal otitis externa): a prospective study. J. laryngolotol, 1989;103:30-5

12. Mohanty JC, Mohanty SK, Sahoo RC, Ghosh SK et al. Clinico microbial profile of Fungal otitis externa in Berhampur. Indian $\mathrm{J}$ of Otology 1999;5(2): 81-83.

13. Prasad SC, Kotigadde S, Manisha S et al. Primary Fungal otitis externa in the Indian Subcontinent: Predisposing Factors, Microbiology and Classification. International $\mathbf{J}$ of Microbiology 2014; (about9p.) Available from:

14. X Jia, LiangQ,Chi $\mathrm{F}$ and Cao W. Fungal otitis externa in Shanghai:Aetiology,Clinical features and Therapy. Mycoses 2012; 55: 404-409.

15. Otomycosis in Adolescent Patients Referred to the Therapeutic Centers in BabolCity, Iran Keivan Kiakojuri' Ramazan Rajabnia, BaharehJalili , Soraya Khafri \& Saeid Mahdavi Omran Jundishapur Journal of Microbiology: May 2015, 8 (5); e17138

16. Pradhan B,Tuladhar NR and Amatya RM. Prevalence of Fungal otitis externa in outpatient department, department of Otolaryngology in Tribhuvan university Teaching Hospital, Kathmandu, Nepal. Ann Otol Rhinol Laryngol 2003;52: 76-80. 